\title{
Relationships between self-esteem and marital satisfaction among women
}

\author{
Zahra Yadalijamaloye ${ }^{1}$, Elham Naseri ${ }^{2}$, Mojgan Shoshtari ${ }^{3}$, Mohamad Khaledian ${ }^{4,}$, \\ Razeye Ahrami ${ }^{5}$ \\ ${ }^{1}$ M.A in Women's Studies, University of Shiraz, Shiraz, IRAN \\ ${ }^{2}$ Factoly of psychology department of Payame Noor University, PO BOX 19395 - 3697 , Tehran, IRAN \\ ${ }^{3}$ Student PhD of Psychology of Children with Special Needs, Payame Noor University, PO BOX 19395 - 3697, Tehran, IRAN \\ ${ }^{4}$ Factoly of psychology department of Payame Noor University, PO BOX 19395 - 3697 , Tehran, IRAN \\ ${ }^{5}$ Department of Psychology and Education of Children with Special Needs, University of Isfahan, Isfahan, IRAN
}

\section{Email address:}

Mohamad_khaledian22@yahoo.com(M. Khaledian)

\section{To cite this article:}

Zahra Yadalijamaloye, Elham Naseri, Mojgan Shoshtari, Mohamad Khaledian, Razeye Ahrami. Relationships between Self-Esteem and Marital Satisfaction among Women. Psychology and Behavioral Sciences. Vol. 2, No. 3, 2013, pp. 124-129.

doi: $10.11648 /$ j.pbs.20130203.17

\begin{abstract}
This study explores relationships between self-esteem and marital satisfaction among women in Najafabad and Fuladshahr in Iran. The case study was 757 women (381 respondents in Najafabad and 376 in Fuladshahr) who were married for at least 3 years. Findings show that there is a significant relation between age of women, marriage duration and marital satisfaction. Also generally the rate of marital satisfaction was higher between Fuladshahr women. Marital satisfaction was found to be positively correlated with self-esteem in both cities, so that higher self-esteem was associated with greater satisfaction. Another finding was that level of marital satisfaction; number of children and duration of the marriage were negatively correlated for women. The result of multiple regression analysis showed that factors including: number of children, marriage duration, the number of children and self-esteem could predict $19.5 \%\left(\mathrm{R}^{2}=19.5\right)$ of dependant variable variations in Najafabad and age of woman, marriage duration, the number of children and self-esteem could explain $0.28 \%\left(\mathrm{R}^{2}=0.28\right)$ of the marital satisfaction among women in Fuladshahr.
\end{abstract}

Keywords: Marital Satisfaction, Self-Esteem, Women, Fuladshahr

\section{Introduction}

One of the important discussions in the family is the quality of the relationship between the parents (husband and wife). When the relation between these two is satisfactory, some sort of comfort prevails over the family, resulting in an improved performance in the social life. One of the effective factors in making this process effective whose outcome is the marital satisfaction is psychological issues. Self-esteem is a psychological variable that plays an imp0ortant role in the lives of people. For this, focusing on the couples' self-esteem could result in increasing their marital satisfaction an improving the families' efficiency in the end. The past studies indicate that the self-esteem is factor that is effective in the women marital satisfaction rate compared to men (Cohen et al, 2009).

Theorists from different disciplines of sociology, psychology, anthropology and etc... have been theorizing about the family structure, while generally speaking, the family structure is a social-psychological one in the sense; both social and psychological factors are effective in it. In other words, each person in this structure is affected by internal (psychological) and external (social) conditions of the family (Aazazi, 1998). As noted, since the concept of the marital satisfaction is an issue associated to the social and psychological field, the theories about the family could be divided into three parts: the first part is about sociological theories (mostly micro theories); the second part involves social and psychological theories and the third part includes psychological theories regarding the family therapy range. Askanzuny, Bott, Beck-Grensheim, Duvall and Hill are among the sociologists who have been theorizing in this area whose theories have mostly been related to the structural characteristics of the nuclear family. Social and psychological theories include: the need theories, the expectation theories, the social learning theories, and 
the social experience theories. The third part of the theories involves psychological theories regarding the family therapy range. Theorists in this area are Kampler, Bowen, and Bowlby. Therefore according to the shortcomings of the theories and existing approaches, it looks the theory of the family life cycle by Duvall and Hill is appropriate for investigating the marital satisfaction because it has been used in numerous researches is approved by many others(Peleg, 2008; Fakhraee, 2010). In the late 1940s, the family sociologists including Evelyn Duvall and Reuben Hill for the first time, trying to explain the existing order in the family life, developed a holistic framework for investigating the family. They postulated the family during its own existence deals with its own function and form changes and this process occurs in an orderly set through stage development. These sociologists concentrated on that category of organizational and adaptive changes which the family must show response to its own structural changes. They had in particular, based their own theoretical model on the changes deriving from the expansion and the smaller family size, changes in the age composition, the age of the oldest child and the changes originating from the job status of people as breadwinners ( Goldenberg, 2010). According to this perspective, despite various compositions and structures, families go through occurrences and specific and predictable stages (e.g. marriage, the birth of the first child, children leaving home, the death of the grandfather and mother). Each stage occurs due to a specific event, which in Zillah's opinion this event is a stage index of the family that requires changes and re-adaptation to that event. These stages could happen because of changes to the compositional structure of the family or happen due to fundamental changes in the self-following process of the members (going to school, entering to the adolescence world, retirement). The family system characteristics at the time of development are continuation and change. In some cases, they said changes are orderly, gradual and continued. In other cases, it is possible for these changes to be sudden, annoying and inconsistent. In both situations it is necessary for the overall system of the organization to undergo changes and developments. For instance, it is possible that a family encounters unexpected and catastrophic occurrences. These crises disrupt the normal developmental process and will inevitably render in communication changes among the members of the family (Goldenberg, 2010).Generally, based on this perspective, family relations become more complex over time while families have different needs and inclinations in different stages of the life cycle. In the early (before becoming parents) there is great joy that decreases in the mid years of the marriage (years of being parents) while it increases in the final years of the marriage (after being parents). Having children reduces the marital satisfaction rate in the early years while as there is less work and the responsibilities of the parents are reduced, these two will increase the marital satisfaction in the final years of the marriage (Peleg, 2008).

The Duvall and Hill's theory provide a useful theoretical framework for explaining the marital satisfaction of the married women residing in Najafabad and Fuladshahr. Based on this theory, the model and the research hypotheses are as follows:

Conceptual model of the research has been selected based on the Duvall and Hill's theory and shows what factors are related with the marital satisfaction.

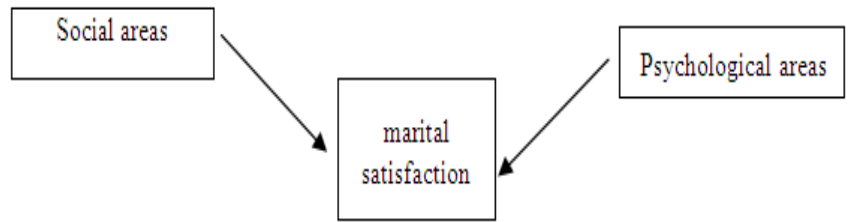

Figure 1. The conceptual model of the research

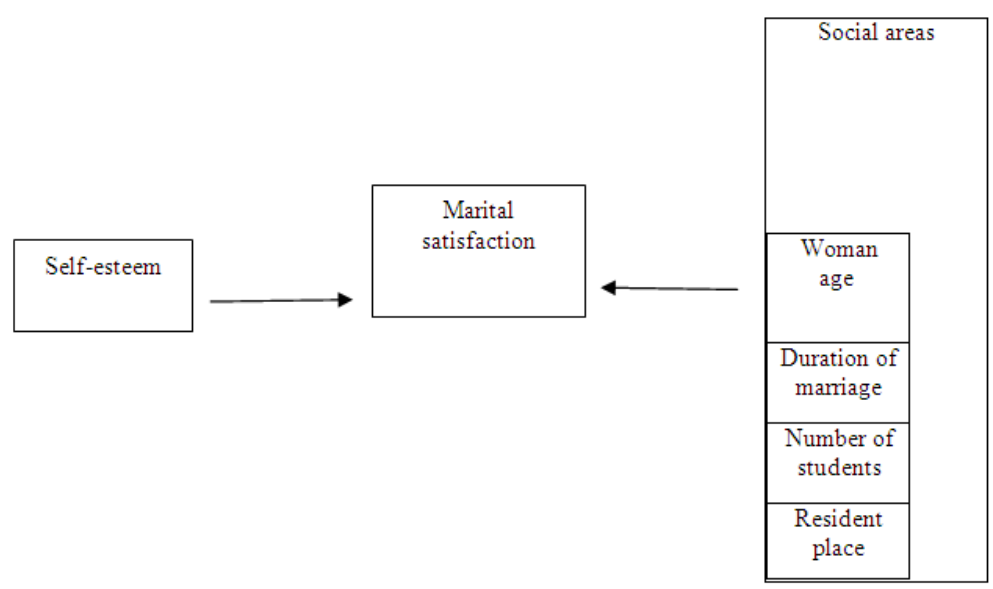

In this section the applied concepts in the theory considered become operational in the research framework

Figure2. The empirical model of the research 
Also, the city (living place) could affect self-esteem and the marital satisfaction of the people. The selection of the cities of Najafabad and Fuladshahr has been due to their different social characteristics, cultural distinctions, religious and demographic properties. Najafabad is a city in the transition from tradition to modernity which has largely maintained its traditional and indigenous composition in its transition period. On the other hand, it has embraced elements of modernity within its own culture. However, Fuladshahr is a city of immigrants which due to its same characteristics does not enjoy a united and consistent culture. Furthermore, the diversity of cultures and different ethnic groups in this city have resulted in positive and negative consequences in the lives of people ion there. Statistics indicate that Fuladshahr compared to Najafabad has a higher number of immigrants. According to the results of the general census of population and housing in 2007 the total population migrated to Najaf Abad equaled to 44,401 persons, while the rate for the city of Fuladshahr was 44,803 people, while Najaf Abad, is a large city whose the entire population equals to 282,430 persons, indicating that the rate of immigration to the population is not that large. However, Fuladshahr is part of the city of Lenjan whose population is 55496 people. Hence, this rate of immigration for Fuladshahr is great. Therefore, the Fuladshahr's population accounts for two-thirds of the immigration while this rate for Najafabad is one-fifth. For this, a comparative investigation of the marital satisfaction in these two cities could explain the impacts of cultural, indigenous composition as well as that of immigration on different aspects of the residents of these two cities including the marital satisfaction aspect. Thus the general objective of this paper is to answer the question as to what relation is there between the self-esteem and marital satisfaction in married women of these two cities that are socially and culturally different.

\section{Research Hypotheses}

1. There is a significant relationship between the city where the women live and their marital satisfaction rate

2. There is a significant relationship between age and their marital satisfaction rate

3. There is a significant relationship between the duration of marriage and their marital satisfaction rate

4. There is a significant relationship between the number of children and their marital satisfaction rate

5. There is a significant relationship between self-esteem and their marital satisfaction rate

\section{Research Methodology}

The method used in this research is based on quantitative methods and survey techniques. In terms of dealing with the approach, a field approach was used. This study collected data through direct talks to the respondents by the researcher and questionnaires submitted to them. The survey questionnaire consisted of two parts. Part one is assigned to variables such as age, number of children and etc.... Part two includes a number of open and closed questions by the researcher himself as well as two spectra under the name of Lee marital index (LMI) for measuring the marital satisfaction and the self-esteem spectrum. Both of these spectra were measured using the Likert scales. The Lee marital questionnaire is made up 5 subscales that includes empathy, sexual satisfaction, doing things together, emotions and relationships, which includes 27 items in the form of the Likert spectrum from totally agree to totally disagree. This measurement tool had already been used in the research by Lee (1997) and its validity has been supported. Prior the administration of it, he also provided some experts with it who had approved its validity.

The statistical population in this research includes all married women residing in the two cities of Fuladshahr and Najafabadin the province of Isfahan who were married for at least 3 years. In this research, each and every married woman is considered a statistical analysis unit in the society under investigation. The research questionnaire were distributed in the two cities of Isfahan and the data were collected in this way. In this research, using the estimation formulae of the sample size, a certain number of married women were selected and became the subject of the study. The Cochran formula was used in here. Based on the sample size and at the significance level of $95 \%$ and the sampling error of 5\%, 376 people for Fuladshahr and 381 people for Najafabad were selected. In total, the sample size of the two cities equaled 757 people. Here, in the research, the sampling methods to obtain a research sample were quota and random sampling. In this case, first in proportion to the household population of each area of both cities, a quota was assigned to each area and then, inside each area the sample participants were selected by the questioners through simple random sampling.

To measure the validity of the questions in the present research, the questionnaire was given to some professors including sociology and psychology professors and their views were applied. Some changes were made wherever necessary in some questions. To translate the Lee marital spectrum, it was translated by some professional translators and having the exact translation was made, it was given to professors for final inspection. To measure the internal consistency of the items, the Cronbach's alpha was applied. When the alpha coefficient value becomes closer to 1 , the items have more internal consistency. Having the reliability measurement of the concepts under study was achieved; values for the Cronbach's alpha were obtained which are presented in the following table, indicating appropriate internal consistency for the questionnaires questions.

Cronbach's alpha rate (reliability) of the items of each spectrum

Composition Number of items Cronbach's alpha




\begin{tabular}{|c|c|c|c|c|c|c|}
\hline $\begin{array}{c}\text { LMI } \\
\text { Self-esteem } \\
\end{array}$ & $\begin{array}{l}27 \\
10 \\
\end{array}$ & $\begin{array}{l}0 / 84 \\
0 / 55 \\
\end{array}$ & \multicolumn{2}{|c|}{ 4. Findings } & & \\
\hline City & \multicolumn{2}{|c|}{ Najafabad } & \multicolumn{2}{|c|}{ Fuladshaahr } & \multicolumn{2}{|c|}{ Total } \\
\hline Marital satisfaction & Frequency & Percentage & Frequency & Percent & Frequency & Percentage \\
\hline Low & 53 & $13 / 9$ & 57 & $15 / 2$ & 110 & $14 / 5$ \\
\hline Average & 262 & $68 / 8$ & 234 & $62 / 2$ & 496 & $65 / 5$ \\
\hline High & 66 & $17 / 4$ & 85 & $22 / 6$ & 151 & $0 / 21$ \\
\hline
\end{tabular}

1. There is a significant relationship between the city where the women live and their marital satisfaction rate.

As noted in table. 2 the hypothesis about the relationship between the city of the respondents and their marital satisfaction rate is supported at the $95 \%$ confidence level. The total average marital satisfaction rate among women in Fuladshahr is higher than those living in Najafabad.

Table 2. the relationship test between the city of the respondents and the marital satisfaction rate

\begin{tabular}{llccc}
\hline Dependant variable & City & Frequency & Average & t \\
\hline The total marital & Najafabad & 381 & $94 / 74$ & $-0 / 739$ \\
satisfaction rate & Fuladshahr & 376 & $95 / 78$ & $0 / 02$ \\
\hline
\end{tabular}

2. There is a significant relationship between age and their marital satisfaction rate.

According to the Pearson coefficient in table .3 which is $-0 / 251$ in Najafabad, $-0 / 309$ in Fuladshahr and $-0 / 283$ in total, the age variable of the respondents is related to their marital satisfaction rate. Negative coefficient means there is an inverse relationship between these two variables. In other words, as the age of women increases the satisfaction decreases. This relationship is significant at least at the $95 \%$ confidence level.

Table 3. The Pearson correlation coefficient between the age of women and their marital satisfaction rate.

\begin{tabular}{|c|c|c|c|}
\hline \multicolumn{2}{|c|}{ Independent variable } & \multirow{2}{*}{$\begin{array}{c}\text { Pearson coefficient } \\
-0 / 251\end{array}$} & \multirow{2}{*}{$\frac{\text { Significance level }}{0 / 001}$} \\
\hline & Najafabad & & \\
\hline \multirow[t]{2}{*}{ Age } & Fuladshahr & $-0 / 309$ & $0 / 001$ \\
\hline & Total & $-0 / 283$ & $0 / 001$ \\
\hline
\end{tabular}

3. There is a significant relationship between the duration of marriage and their marital satisfaction rate.

According to the Pearson correlation coefficient in table. 4 which in Najafabad, Fualdshahr, and in total are -0/256, $0 / 321$ and $0 / 289$ at the significance level of $0 / 000$, the relationship between the duration of the marriage and the marital satisfaction at the $95 \%$ confidence level is significant. The Pearson correlation coefficient is negative in the sense as the length of marriage increases the satisfaction rate of the women decreases.

Table.4. the Pearson correlation coefficient between the duration of marriage and the marital satisfaction of the respondents

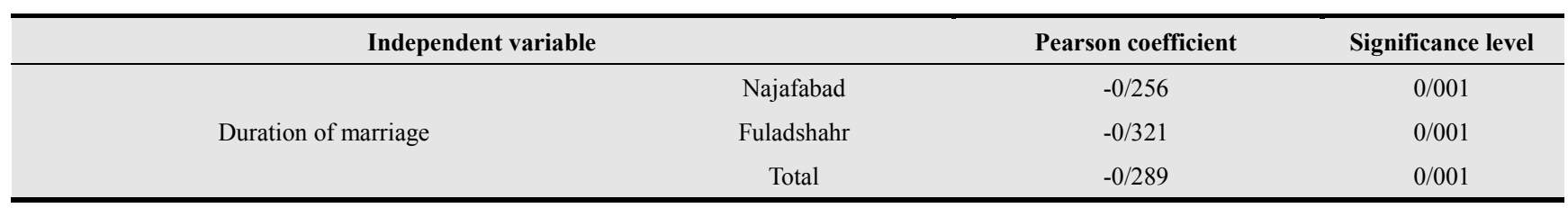

4. There is a significant relationship between the number of children and their marital satisfaction rate.

According to the obtained correlation coefficient in the following table which is $-0 / 281,-0 / 227,0 / 254$. It is concluded the relationship between the number of children and the women marital satisfaction is inverse. In other words, as the number of children increases, the satisfaction rate decreases, hence this relationship is at the $95 \%$ confidence level significant.

Table 5. The Pearson correlation coefficient between the number of children and the satisfaction rate

\begin{tabular}{lccc}
\hline \multicolumn{2}{c}{ Independent variable } & Pearson coefficient & Significance level \\
\hline & Najafabad & $-0 / 281$ & $0 / 001$ \\
\multirow{3}{*}{ Number of children } & Fuladshahr & $-0 / 227$ & $0 / 001$ \\
& Total & $-0 / 254$ & $0 / 001$ \\
\hline
\end{tabular}


5. There is a significant relationship between self-esteem and their marital satisfaction rate

There is a significant relationship between self-esteem and their marital satisfaction rate in terms of the city residents live. The Pearson correlation coefficient in (table 6) indicates a direct relationship between the two variables of self-esteem and the respondents' marital satisfaction rate in Najafabad, Fuladshahr and in general. In other words, as the self-esteem increases among the women, the satisfaction rate increases. This relationship is at the $95 \%$ confidence level significant.

Table 6. The Pearson correlation coefficient between the self-esteem and the marital satisfaction women

\begin{tabular}{cccc}
\hline & Independent variable & Pearson coefficient & Significance level \\
\hline & Najafabad & $0 / 350$ & $0 / 001$ \\
Self -esteem & Fuladshahr & $0 / 459$ & $0 / 001$ \\
& Total & $0 / 770$ & $0 / 001$ \\
\hline
\end{tabular}

6. Levels of explaining the marital satisfaction in Najafabad and Fuladshahr

The multiple regression test was conducted for each city separately. Table.7.has been assigned step by step multivariable analysis for predicting the dependant variable of the (total) marital satisfaction in Najafabad. In this city, the four variables of self-esteem, number of children, duration of marriage and the age of women have explained the $19 / 5 \%$ of the dependant variable variations of the (total) marital satisfaction. The negative Beta rate in the variables of the number of children, duration of marriage and the age of the woman indicate an inverse impact of these variables on the marital satisfaction .

Table 7. Step by step multivariable analysis for predicting the dependant variable of the (total) marital satisfaction in Najafabad

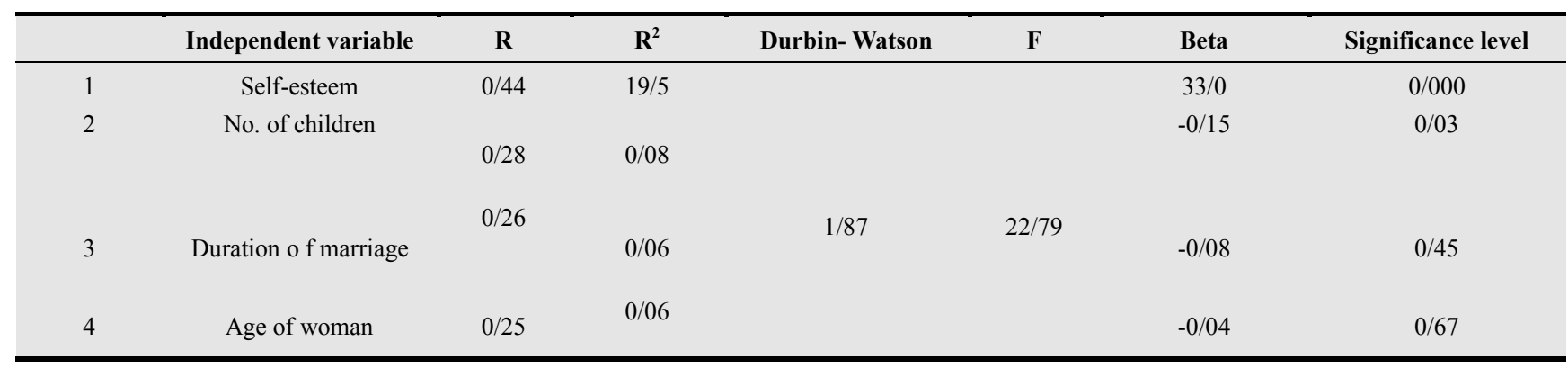

Table8. has been assigned step by step multivariable analysis for predicting the dependant variable of the (total) marital satisfaction in Fuladshahr. In this city, the four variables of self-esteem, number of children, duration of marriage and the age of women have explained the $28 \%$ of the dependant variable variations of the (total) marital satisfaction. The Beta value in the woman age and the duration of the marriage is negative. In other words, as the age and the length of marriage in women of Fuladshahr increases, the satisfaction rate among them decreases.

Table 8. Step by step multivariable analysis for predicting the dependant variable of the (total) marital satisfaction in Fuladshahr

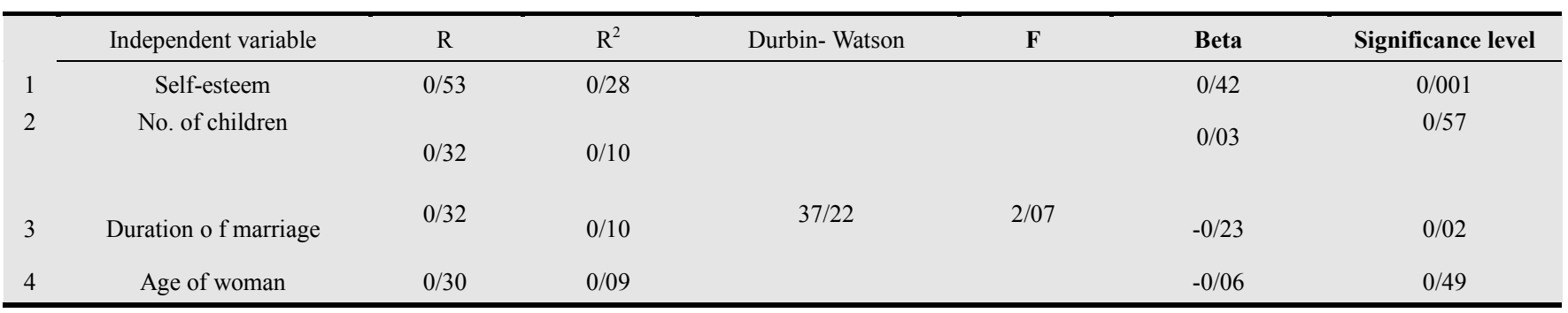

\section{Discussion}

The performed research aims to investigate the impact of self-esteem on the women marital satisfaction rate regarding the city they live in. As noted, the results of the research were presented in two inferential and descriptive statistics sections. In the descriptive section, the results indicate that most women were in their adult and young years. The average duration of marriage of the women was 14 years and the average number of children was 2 people. Women in Fuladshahr were generally having more marital satisfaction. In terms of psychological; well-being, the women respondents were in an average level. However, in the second section the following hypotheses were achieved:

First hypothesis: There is a significant relationship between the city where the women live and their marital satisfaction rate; As noted, this hypothesis was supported at 
least at the $95 \%$ confidence level. Generally, the total average marital satisfaction rate among women in Fuladshahr was higher than those of Najafabad .Hence, it was determined that the city influences on the marital satisfaction rate among women. Second hypothesis: There is a significant relationship between age and their marital satisfaction rate; The hypothesis was supported at the $95 \%$ confidence level. The results of this test conforms with the results of a research by Peleg(2008) in connection with the fact that as the age of women increases, their marital satisfaction rate decreases. The result also supports the perspective from which this hypothesis has been taken. Research by Majidi also confirms his hypothesis. Third hypothesis: There is a significant relationship between the duration of marriage and their marital satisfaction rate; The hypothesis is too confirmed. It was also determined the results of testing this hypothesis correspond with the results of the research by Peleg(2008), Fakhraee(2010) and Majidi( 2007) which have all originated from the life cycle perspective. Fourth hypothesis: There is a significant relationship between the number of children and their marital satisfaction rate; the hypothesis was supported at the $95 \%$ confidence level. The result of this test conforms to the results of a research by Peleg (2008). Fifth hypothesis : There is a significant relationship between self-esteem and their marital satisfaction rate; The hypothesis is too confirmed. The highest marital satisfaction was related to women in Fuladshahr who had higher self-esteem. The lowest rate of satisfaction was related to women in Najafabad. Therefore, as self-esteem increases, women marital satisfaction rate also increases. Shafiee Kanjani (2008), Cohen et al (2009), Butzer and Campbell (2008), Hollist et al(2007), and Majidi ( 2007) have in their own researches affirmed the significant relationship between the two variables.

Step by step multivariable analysis for predicting the dependant variable of the (total) marital satisfaction in Najafabad showed the two variables of number of children and the psychological well-being have explained $19 / 5 \%$ of the dependant variable of the (total) marital satisfaction. As noted in this analysis, the importance of this psychological variable in this research namely self -esteem is quite evident in predicting the dependant variable. Thus, improving self-esteem results in an increased marital satisfaction. Predicting the dependant variable of the (total) marital satisfaction in Fuladshahr indicated the two variables of the women age and psychological well-being could explain $28 \%$ of the dependant variable variations in Fuladshahr. The general results of the research showed as the age and the number of children increase, rate of satisfaction decreases. As self-esteem increases, rate of satisfaction increases. Thus, improving self-esteem increases the marital satisfaction of the women.

\section{References}

[1] Aazazi, Sh. (1998). Sociology of Family with the emphasis on the role, structure and function of it in the contemporaries, Tehran: Roshangaran publication.

[2] Shafieekanjani, A. R., Mahyar, A., Janbozorgi, M., Noohi, S., \& Hosseini, S. R. (2008). Investigating the psychopathology correlation of women and marital satisfaction, Pejoohande monthly periodical, 6: 483-490.

[3] Fakhraee, S. (2010). A sociological explanation of couples conflicts in Shiraz Housholds. PhD thesis at Shiraz University, Department of Sociology.

[4] Goldenberg, I., \& Goldenberg, H. (2010). Family therapy. (trans.by Hamid Reza HosseinShahiBaravati., Syamak Naghshbandi., \& Elham Arjomand) .Tehran: Ravan Publication.

[5] Majidi, M. (2007). Investigating social-economic factors related to the marital satisfaction of the immigrant women in oil rich areas of the south. M.A Thesis of the Shiraz University.

[6] Butzer, B., \& Campbell, L. (2008)." Adult attachment,sexual satisfaction and relationship satisfaction:a study of married couples,personal relationships".Personal relationships. 15: $141-154$

[7] Cohen, O., Geron, Y., \& Farchi, A. (2009)."Marital quality and global well-being among older adult Israeli couples in enduring marriages". The American journal of family therapy.37: 299-317.

[8] Hollist, S., Cody,B., Miller, R., Falceto, O., Fernandes, C., \& Carmen L. (2007)."Marital satisfaction and depression:a replication of the marital discord model in a latino sample",Family process. 46(4): 485-498.

[9] Lee, S. (1999). Marital Satisfaction factors for KoreanAmericans. PH.D dissertation of the school of education of Andrews University, U.S.A.

[10] Peleg, O.(2008)."The relation between differentiation of self and marital satisfaction: what can be learned from married people over the course of life?".The american journal of family therapy. 36 : 388-401. 